MATEC Web of Conferences 35, 03005 (2015)

DOI: $10.1051 /$ matec conf/ 20153503005

(c) Owned by the authors, published by EDP Sciences, 2015

\title{
Detection system of fasteners state based on zigBee networks
}

\author{
Jun-juan Zhao ${ }^{1, \text { a }}$, Bo Wang ${ }^{2}$, Wen-qiang Niu ${ }^{2}$, Xian-hui LI ${ }^{1}$, Bin Zhang ${ }^{1}$ and Yue-yue Wang ${ }^{1}$ \\ ${ }^{1}$ Municipal Key Laboratory of Environmental noise and vibration,Beijing Municipal Institute of Labour Protection, Beijing 100054, China; \\ ${ }^{2}$ Zhe Jiang Tian Tie Industry CO.,LTD, Tian Tai, Zhe Jiang, CHINA
}

\begin{abstract}
The safety requirements demanded from rail traffic have risen above all in view of the rapid development of modern high-speed trains. Railway fastener is the important part of rail line. The normal state of the fastener is the guarantee of the rail transportation security. The missing or broken fastener may be the tremendous threaten to the security, or even cause the serious traffic accidents. This paper presents a new fully automatic and configurable wireless sensor network system able to detect the absence of the fastening bolts that fix the rails to the sleepers. The experement indicated that the system by using sensor networks and LabVIEW can guarantee a high accurate detection for the missing or broken fastening elements in combination with the strain measurement of the rubber pad under rail.
\end{abstract}

\section{Introduction}

Organization the railway maintenance is a particular application context required in order to prevent any dangerous situation [1-4]. With the growing of the highspeed railway traffic, automatic inspection systems able to detect rail defects, sleepers' anomalies [5,6], as well as missing fastening elements, become strategic since they could increase the ability in the detection of defects and reduce the inspection time in order to guarantee more frequent maintenance of the railway network [7-8].

Railway fastener is the important part of rail line. The normal state of the fastener is the guarantee of the rail transportation security. The missing or broken fastener may be the tremendous threaten to the security, or even cause the serious traffic accidents. The detection of the railway fastener state in our country those days is still in the mariner of the manual detection. But this manner of detection is inefficiency and lack of objectivity, because it relies on the worker's technical proficiency. With the development of railway transportation in our country to high-speed and heavy-load, the railway defects including fastener defects grow in number and the manual detection can't meet the needs of modern railway transportation safety. Therefore, the automatic detection of railway fastener state is the problem that the railway development has to face.

In this paper we have developed a new system based on Zigbee Wireless Sensor Networks (WSN), strain measurement of the rubber pad under rail and LabVIEW for detection of the rail fasteners state. As the rubber plate under rail, which directly fastened together with the rail by rail fastener, has alternating strain in the work. This actual alternating strain can well reflect the state of the rail fastener. The initial examinations in the paper indicate the system is especially useful for the state detection of the rail fastener.

\section{Simulations}

In this paper, strain gauge was arranged on the rubber plate under rail to detection of damaged or missed rail fasteners. As the position of the strain gauge arrangement is very important for the detection accuracy, the rubber plate stress and strain state was simulated and analyzed, as shown in the Fig. 1 below.

The maximum strain is at position $\mathrm{A}$, minimum is at position $\mathrm{B}$, the strain at position $\mathrm{C}$ is between $\mathrm{A}$ and $\mathrm{B}$. Considering strain value, installation convenience and the size of the strain gauge, the best position strain gauge arranged is at $\mathrm{C}$.

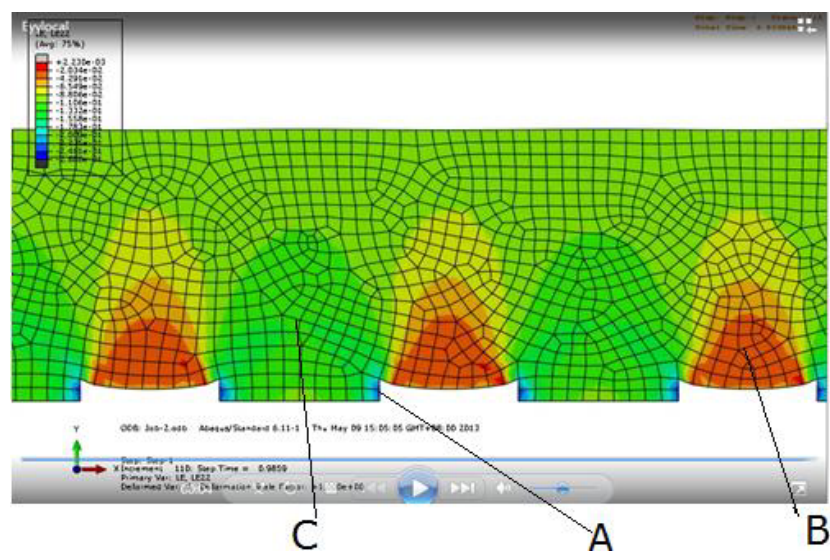

Figure 1. The vertical strain of the rubber plate under the same direction pressure

$\bar{a}$ Corresponding author: junjuanzhao@sina.com 


\section{System design}

This detection system has two parts, one is the wireless sensor network (WSN) base on ZigBee, and one is the data process module (computer and software), shown in Fig.2. Computer with its serial port which can acquire signals wirelessly from WSN; the signal process software designed by LabVIEW, which is the most popular in developing virtual instrument nowadays, and has the following advantages: the flexible arithmetic, updatable software, extensive application, low cost and friendly user interface.

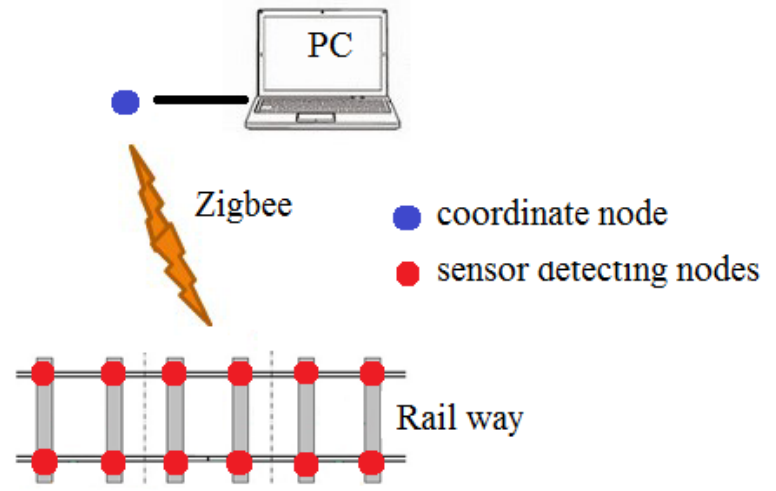

Figure 2. The overview of fastener State detection system based on ZigBee and LabVIEW

\subsection{ZigBee Wireless sensor network}

Wireless Sensor Network (WSN), composed of hundreds of nodes which have ability of sensing, actuation and communicating, has great advantages in terms of high accuracy, fault tolerance, flexibility, cost, autonomy and robustness compared to wired ones. Moreover, it is poised to become a very significant enabling technology in many sectors, so it is widely used in military, environment, health, home and other commercial areas.

In this system, the sensor used in WSN is strain gauge (BHF350-3AA) which is arranged on the rubber plate (Fig.1) under rail to detection of damaged or missed rail fasteners. The ZigBee network layer is in a star topology made up by one sink node (coordinator) and many sensor nodes. When sensors collect strain signals real time, these data will be offered to computer through wireless sensor communicating node.

The ZigBee protocol chips chose isCC2530, which integrated the CPU, RF transceiver, net protocol and the RAM together. CC2530 uses an 8 bit MCU (8051), and has $128 \mathrm{~KB}$ programmable flash memory and $8 \mathrm{~KB}$ RAM. It also includes A/D converter, some Timers, AES128 Coprocessor, Watchdog Timer, $32 \mathrm{~K}$ crystal Sleep mode Timer, Power On Reset, Brown Out Detection and 21 I/Os. Based on the chips, many modules for the ZigBee protocol is provided. And the ZigBee transfer unit could be easily designed based on the modules.
The data process module includes computer and data process software. Computer acquires signals wirelessly from WSN with its serial port and processes data through software. the software designed with LabVIEW, which is the most popular in developing virtual instrument nowadays, has the functions of signals display, Fasteners state analysis and warning, etc.

Especially, the process module has the following advantages: the model hardware, the flexible arithmetic, updatable software, extensive application, low cost and friendly user interface (Fig. 3 is the front interface and its program).
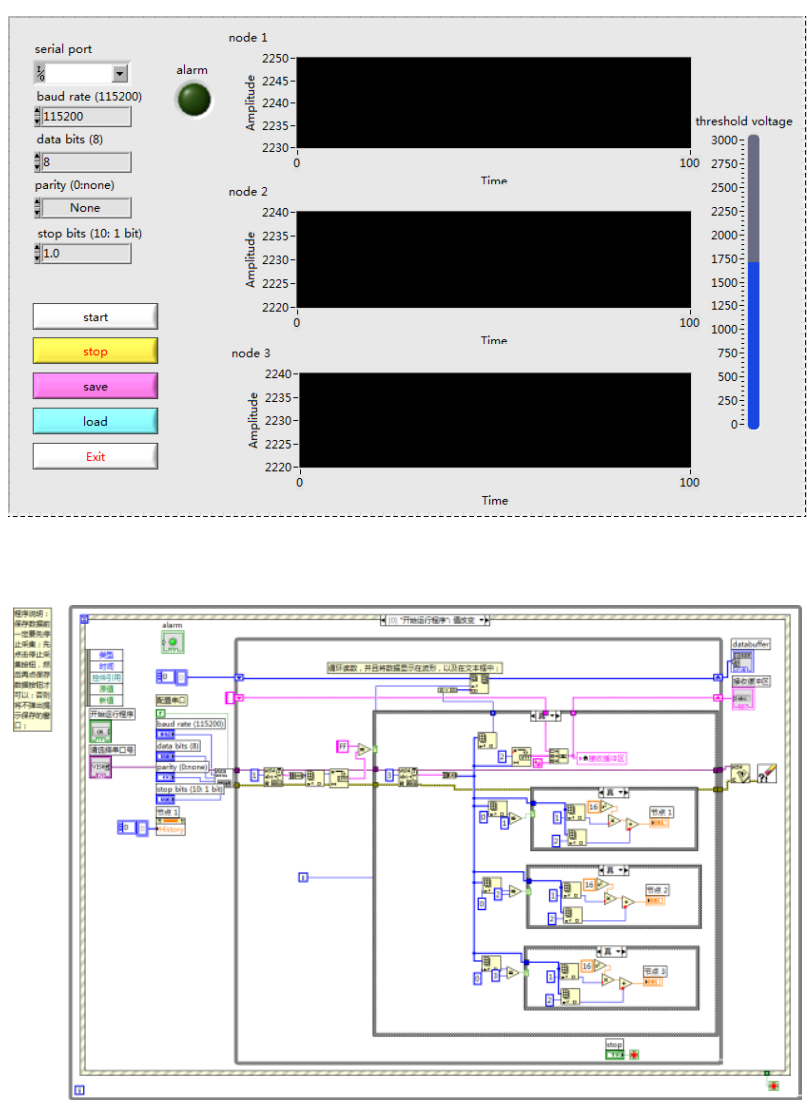

Figure 3. The front interface and the program

\section{Experiments}

In this project, one sensor node was deployed for one fastener, and four nodes were used in this experiment. Through these deployed sensors, the strain information of the fastener can be detected real time. Fig.4 shows the test site of the experiment and the position of strain gauge was deployed.

The test results from the sensor node 1 to 4 shown as from Fig.5, demonstrated a linear relationship between the strain voltage and tightness of fasteners, the tighter the fastener, the smaller the strain voltage, which can be used to judge fasteners state.

\subsection{Data process module}



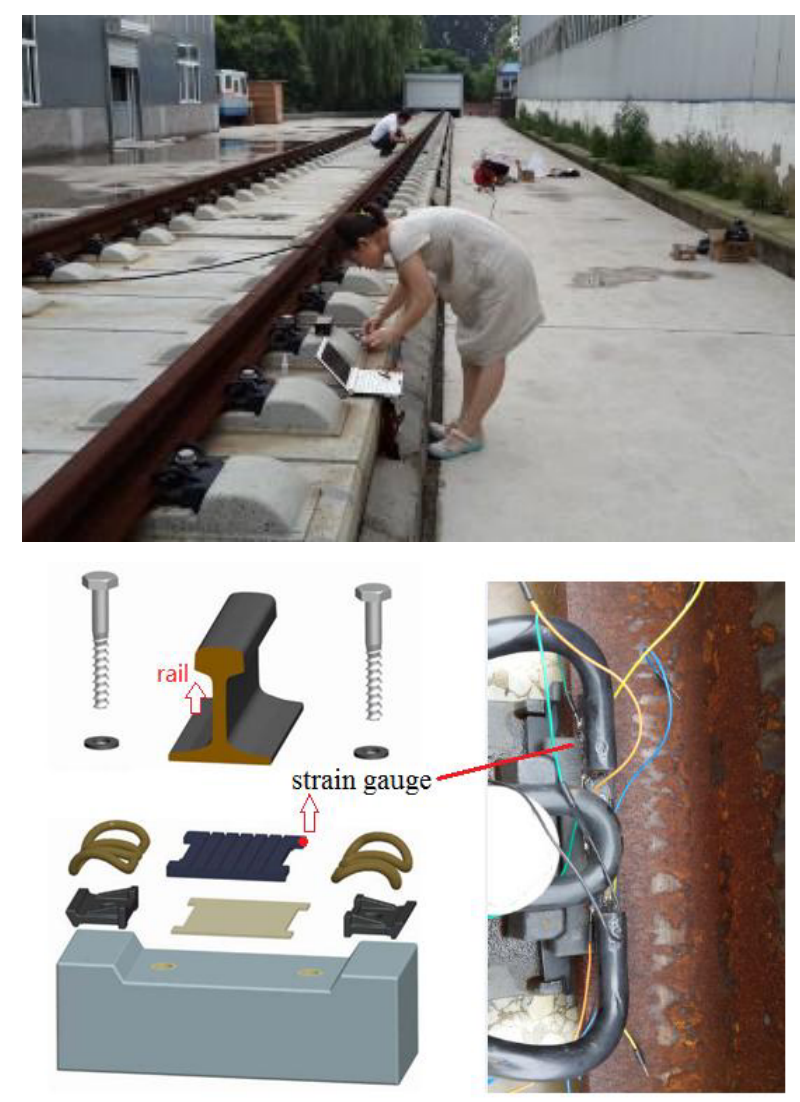

Figure 4. The test site of the experiment and the position of the strain gauge

\section{Conclusions}

This paper presents a wireless sensor network project based on ZigBee technology to be used in the railway fastener state monitoring, which taking CC2530 platform as the main controlling chip, BHF350-3AA as the sensor of strain measurement of the rubber pad. Through the theoretical analysis and experimental test, it has been confirmed that the design of the railway fastener state monitoring system is reasonable, the hardware system performance normal, and the software design is userfriendly that the data can be analyzed and monitored from anywhere with sensors. In combination with the strain gauge arranged on the rubber pad under rail, the strain voltage value as evaluation index guarantee a high accurate detection for the missing or broken fastening elements. The experimental tests have proved that the WSN monitoring system was stable and credible, with the advantages of simple networking, low cost and good maintainability.
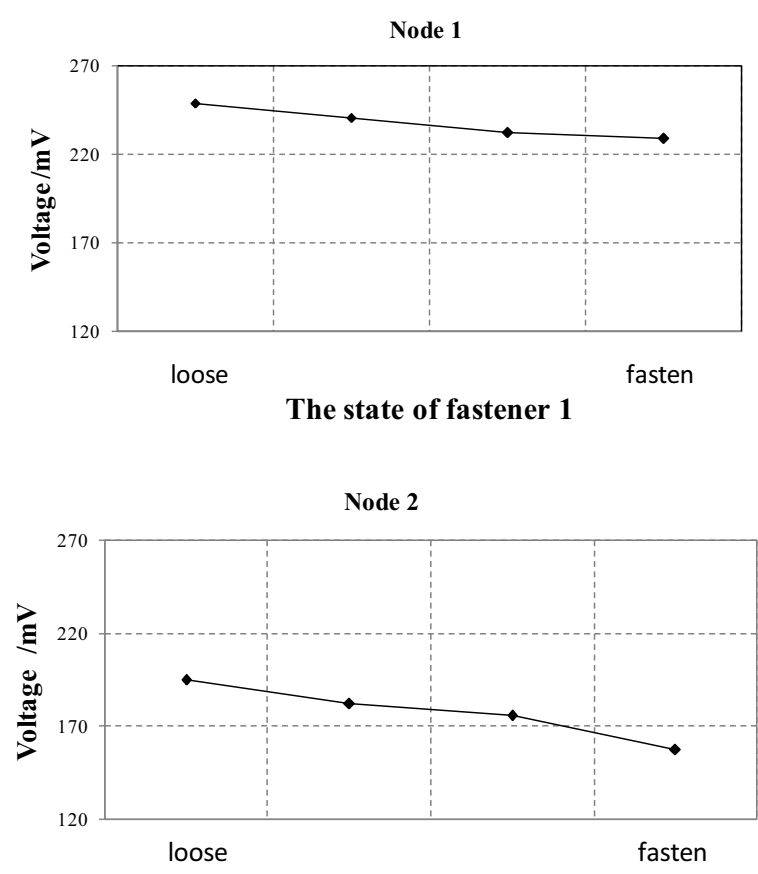

The state of fastener 2
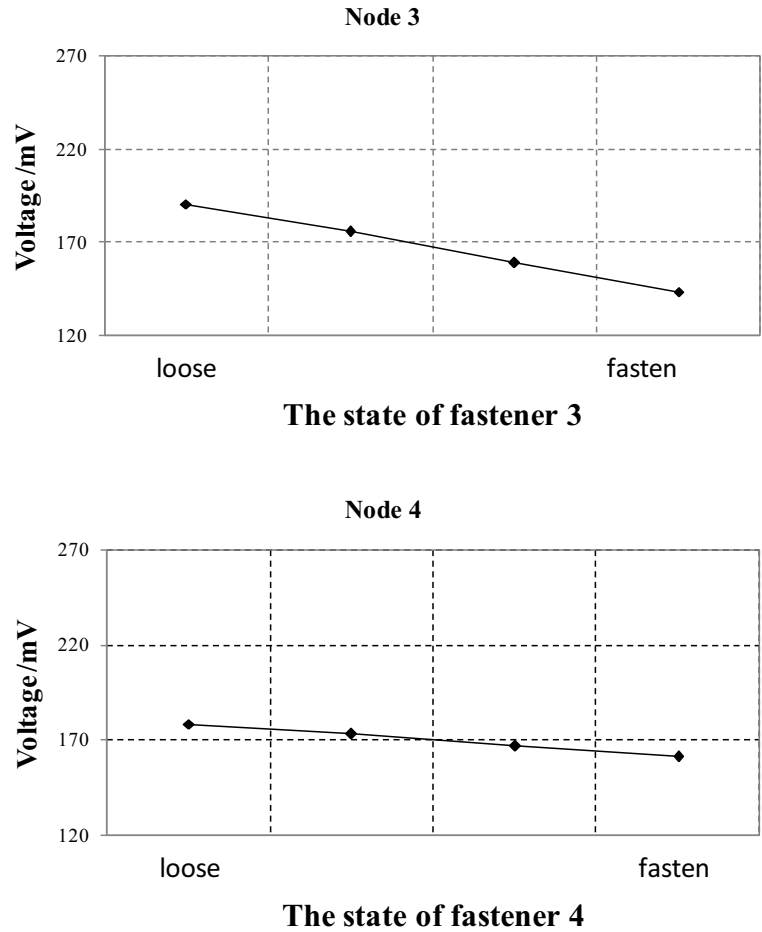

Figure 5. The test results from the sensor node 1 to 4

\section{References}

1. M. Miyamoto, QR of RTRI, 37(1996) : 147-155.

2. J. A. Elkins, H. Wu, Vehicle System Dynamics Supplement, 33(1999) : 293-305.

3. A. Kalmel, L. M. Sweet, Transactions of ASME, 51(1984):680-686.

4. T. Miyamoto, H. Ishida, M. Matsuo, QR of RTRI, 38(1997) : 117-122.

5. X. Jin, P. Wu, Z. Wen, Wear, 253(2002) : 247-256. 
6. W. M. Zhai, C. B.Cai, S. Z.Guo, Vehicle System Dynamics, 26(1996) : 61-79.

7. P. L.Mazzeo, M.Nitti, E.Stella, A.Distante, Pattern Recognition Letters, 25(2004) :669-677.

8. S.Yela, M.Dougherty, N. K.Gupta, Transportation Research Part C, (2008). 